osis A. The cell body swells, the processes are withdrawn and the cytoplasm is reduced to a coarse mesh. The nucleues is compressed by the surrounding fat droplets. By the gold method, the transmittal cells which have taken up a large amount of vitamin A are more intensely impregnated with gold than in the normal condition. This finding suggests the mechanism of the gold method with the aid of in vitro test for the reaction of gold chloride to vita. min A ester. In the hepatic cells vitamin A fluorescence is also demonstrable in fat droplets arranged on the cell border adjucent to the sinusoids. Whereas, the Kupffer cells of the reticulo-endothelial system fail to show any vitamin A uptake. When a rabbit is injected subcutaneously with vitamin A and intravenously with Indian ink, the former is taken up by the transmittal cells, while the latter is deposited as carbon particles in the Kupffer cells. Electron microscopically, the striking finding is that the cell filled with masses of lipid are situated outside of the lining of the hepatic sinusoids and are definitely different from the Kupffer cells.

The present findings may shed another light to the possible significance of vitamin $\mathrm{A}$ for the autonomic nervous periphery.

\title{
References
}

1) Hirt, A. u. K. Wimmer (1939) Klin. Wochschrf., 18:733. $\quad$ 2) Kupffer, C. (1876) Arch. f. mikr. Anat., 12: 352. 3 3) Luft, J.H. (1961) J. Biochem. Biophysic. Cytol. 9:409. 4) Popper, H. (1944) Physiol. Rev. 24:205. $\quad$ 5) Popper, H. and F. Schaffner (1957) Liver: Structure and function. McGraw-Hill, New York. $\quad$ 6) Suzuki, K. (1958) The experimental therapy. Vol. 310-320. 7) Suzuki, K. (1963) Proc. of the 16th general assembly of the Japan medical congress. April, 1963, Osaka. IV : $13 . \quad$ 8) Tomita, Y. (1955) J. Kyoto Pref. Med. Univ. $57: 489$. 9) Watanabe, S. (1960) J. Osaka City Med. Center 9 (Suppl. 8) : 99.

\section{A Histochemical Study of Proliferated Bile Ductules in Viral Hepatitis and Posthepatitis Cirrhosis}

\author{
Kiyowo Kosaka, Yasuyuki Ohta, Hiroshi Endo, \\ Shunji KinUgasa, and Akio Orime
}

The First Department of Internal Medicine, Okayama University Medical School, Okayama

Ductural cell proliferation is thought to be a reaction of the liver against injury. It is one of the parenchymal cell reactions appeared in various pathologic conditions in combination with the mesenchymal cell reactions such as Kupffer cell activation. Recently, rather selective proliferation of the cholangiole cell itself was produced by feeding the rat with $\alpha$-naphthyl isothiocyanate ${ }^{12}$. However, primary hepatic cell injury caused by usual hepatotoxic agents and 
infections also induced ductural cell proliferation as well when the injury lasted continuously. The origin and nature of such proliferated bile ductule is, however, only poorly understood to date. The purpose of the present paper is to describe succinic dehydrogenase activity of the proliferated ductular cells and to discuss the origin and nature of them according to the results.

\section{Material and Method}

Needle liver biopsy specimens of 39 cases of liver diseases; chronic viral hepatitis in 19, cholangiolitic hepatitis in 1 , liver cirrhosis in 19 were taken from the right lobe of patient livers under the vision of peritoneoscopy. The tissue was divided into two pieces and fixed one part in alcoholic Bouin solution for hematoxylin-eosin, PAS, and Azan stain. Another part was frozen in cold isopentane maintained at $-80^{\circ} \mathrm{C}$ for cryostat section. Histochemical visualization of succinic dehydrogenase ( $\mathrm{SDH}$ ) was performed by the method of Nachlas ${ }^{2)}$. Menadione and phenazine methosulphate was individually added in the reaction mixture producing a ratio of $20: 1$ as an intermediator ${ }^{3)}$. In selected cases, Regaud's mitochondria stain were performed. The serial sections, $12 \mu$ thickness each, were cut for reconstruction of proliferated bile ducts and ductules in a case of postnecrotic liver cirrhosis. The Abbe's apparatus was used for the drawing of tissue structure on a clear vinyl plate.

\section{Results}

\section{Chronic viral hepatitis}

Proliferation of bile ducts and ductules in chronic viral hepatitis was observed to various extent according to the grade of parenchymal cell destruction, preferably in the lobular periphery. An ductular structure consisted of cuboid cells smaller than hepatic cells with scanty protoplasma are observed between hepatic columna and smallest bile ducts in the portal triad. Loss of the limiting plate and increased portal space are common underlying histological change in such cases. Histochemically, they possessed moderately strong SDH activity, different from both hepatic cells and bile ducts. Increased formation of formazan granules colored violet blue was observed though they were unable to discriminate each other. The fate of once proliferated bile ductules in the regression was not clearly followed. It seemed to disappear eventually.

\section{Cholangiolitic hepatitis of probable viral origin}

Prolonged and sometimes reccurent jaundice of intrahepatic obstructive nature is characteristic picture of this entity. As shown in Fig. 1, notable proliferation of bile ductules associated with inflammatory cells mainly cosisted of monocyte and lymphocytes is predominant histological findings. The cytoplasm of proliferated ductular cells appeared more eosinophilic and contained more non-glycogenic PAS positive substance than hepatic cells. Histochemically, they are only the ductules of low SDH activity. The cells with the different SDH activity was not observed in them. While a reduction of the 
enzyme activity was observed in the hepatic cells stained with bile pigments (Fig. 2). Such cells usually located in the lobular edge. Scanty of scarcely appreciable mitochondria were stained in the proliferating ductular cells by the Regaud's procedure (Fig. 3).

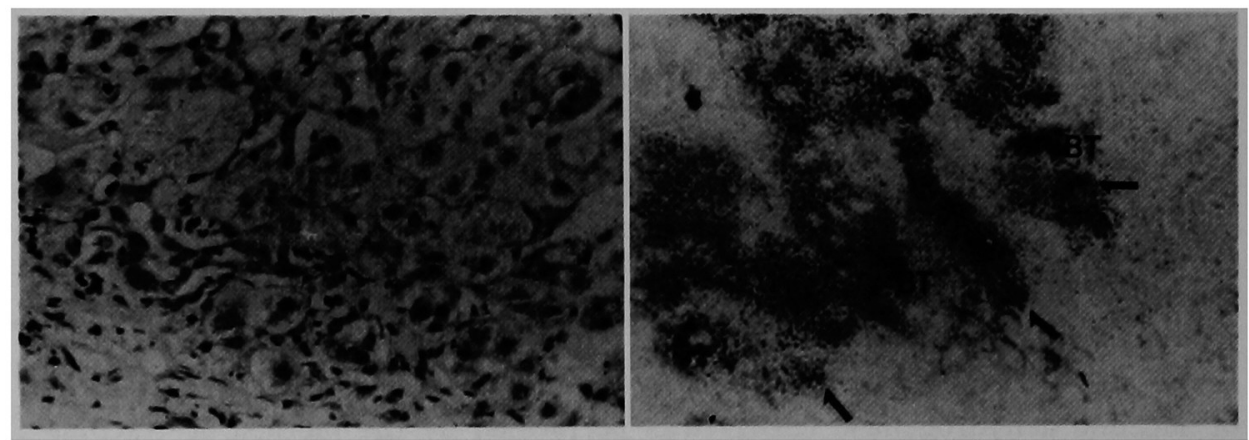

Fig. 1

Fig. 2

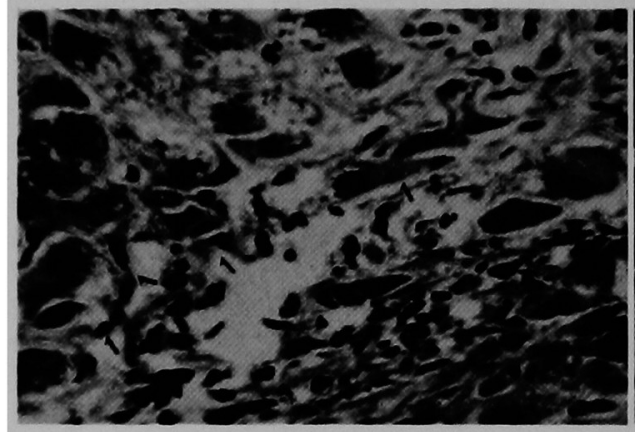

Fig. 3

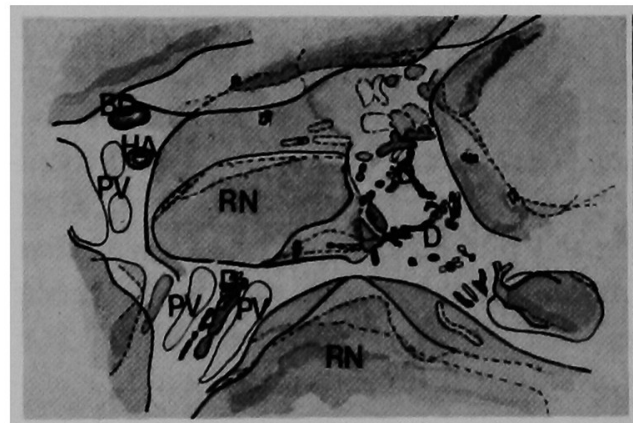

Fig. 5

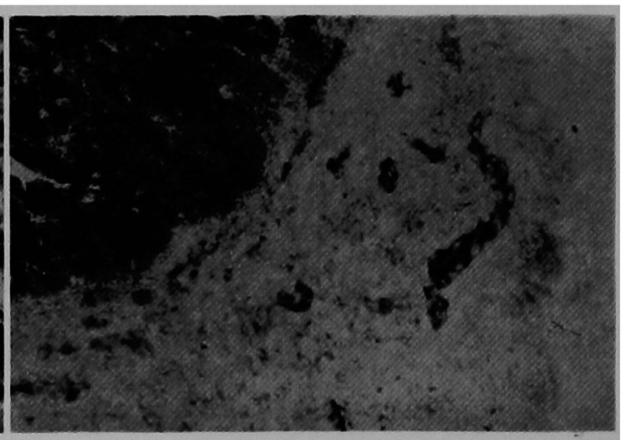

Fig. 4

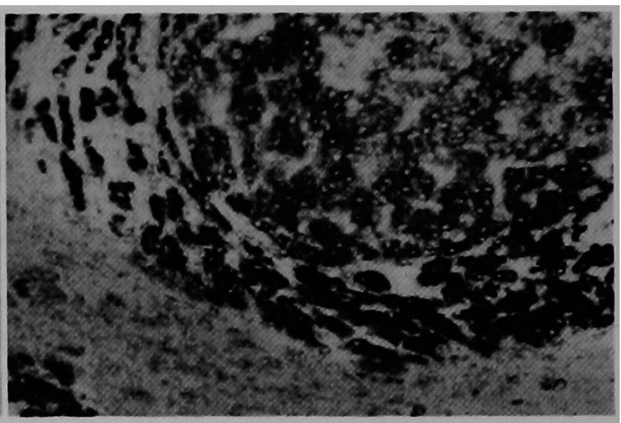

Fig. 6

\section{Liver cirrhosis}

Moderate to marked proliferation of bile ducts and ductules occurs in both portal and postnecrotic cirrhosis. The extent is more intense in the latter. There is an increase in the number of small or interlobular ducts that have lumina as small as 15 microns. Simultaneously, it was observed notable proliferation of bile ductules which have no visible lumina but consisting of cuboid cells with the nuclei being located in the center of the cells. The SDH activity 
of the proliferated ductules was usually as low as those of the small andmedium (trabecular) bile ducts. Occasionally, the ductule consisting of the cells with moderately intense SDH activity was noted within the broad connective tissue. As shown in Fig. 4, one side of such ductule was composed of the cells with low SDH activity and have no glycogen granules, consistent with normal ductular cells. On the contrary, the other portion of the ductule possessed coarse, different sized diformazan granules preferably located in both side of nucleus along long axis and have a number of glycogen granules, consistent with the nature of hepatic cells. Reconstruction study of the ductules elicited that they have connection with small mass of hepatic cells that were thought to be devoid of destruction (Fig. 5). A possibility of development of such cell mass to a regenerative nodule has been suggested", however, it was not encountered in our study. Occasionally, a cluster of cells having homogenous strong activity of SDH were found to encircle the regenerative nodule (Fig. 6). They were not a portion of adjacent regenerative nodule since their circular location around the nodule. Histologically, the cluster of cells were consisted of rather small elongated hepatic cells with eosinophilic protoplasma resulting from expansive development of the regenerative nodule. The connection of bile canaliculi of the cells with the smallest bile ducts was obscure.

\section{Comments}

Proliferating bile ductule with postive SDH activity invariably increased in chronic viral hepatitis and cirrhosis, notably in postnecrotic cirrhosis. Increased formazan formation of ductular cells is thought to be due for the most part to the enzyme contained in the mitochondria of the cells despite a little controversy still exists among electronmiroscopists ${ }^{5,6)}$. Lipids and mucin supposed to be contained in the cells may give a false positive reaction when a tetrazolium salt was used for the enzyme histochemistry. The formazan of Nitro-BT explained to be completely insoluble in those lipids normally found in the tissue ${ }^{\text {") }}$. Besides, cold acetone treatment for the purpose of lipid extraction before histochemical staining procedure gave same results. As to false positive results to mucin, $\mathrm{pH}$ of the reaction mixture was far from the optimum $\mathrm{pH}$ to mucin stain, specified to be 9 to $11^{\mathrm{B})}$.

The SDH activity of the proliferated ductule in cholangiolitic hepatitis was as low as small and medium bile ducts. The results strongly suggested that ductular cell reaction occured primarily and was not the subsequent proliferative change.

Regenerative proliferation of the parenchymal cells in the cirrhotic liver has been considered to occur in the mode of both nodular hyperplasia from preexisting hepatic cells and of transformation to hepatic cells from proliferated bile ductules. The latter has disputed ${ }^{9}$, however, still it reserves special considerations. The possible origin of such proliferated ductules was from degenerating hepatic cells trapped in the surrounding connective tissue and subsequently regenerated two-cell thick plates. The other type of the change may 
occur from the preexisting ductules and smallest bile ducts which are thought to be resistant to injury. The present investigation showed that these two types of ductular proliferation occurs simultaneously according to the extent and mode of parenchymal tissue destruction. The term of pseudotubuli or pseudo-bile ductule ${ }^{102}$ is assumed to be consistent with the proliferated ductular structure originated from degenerating hepatic cells and subsquently regenerating cells. The SDH activity of the cells vizualized by histochemical procedure may provide a differentiation of such ductular cells from the proliferated ductule or preexisting bile ductules and ducts.

As for the cells arranged in circular fashion around the regenerative nodule were considered to be isolated hepatic cells trapped in the surrounding connective tissue in juvenile liver cirrhosis. By the expansive effect of the nodular hyperplasia the trapped cells were compressed and subsequently resulted in condensation of microorganella including mitochondria within the cytoplasma.

\section{Summary}

Proliferated bile ductules in chronic viral hepatitis and subsequent postnecrotic cirrhosis was invariably observed as a regenerative reaction of the liver to injury. The extent of the ductular reaction was proportional to hepatic cell destruction. Histochemical visualization of succinic dehydrogenase was useful for the differentiation of the proliferated bile ductules originated from the trapped degenerative hepatic cells in connective tissue. Thus the term of pseudotubuli may be used with an authentic finding.

The nature and origin of the proliferated bile ductules in cholangiolotic hepatitis seemed somewhat different from that of chronic active hepatitis.

\section{References}

1) Lopez, M. and Mazzanti, L.: J. Path. \& Bact. $69: 243,1955 . \quad$ 2) Nachlas, M.M., Tsou, K.C., DeSouza, E., Cheng, C.S. and Seligman, A.M.:J. Histochem. Cytochem. 5:420, 1957.

3) Wattenberg, L.W. and Leong, J.L.: J. Histochem. Cytochem. 8:296, 1960 . 4) Popper, H. and Schaffner, F. : Liver : Structure and function. New York, Blakiston, 1957, p. 272. 5) Steiner, J.W. and Carruthers, J.S.: Am. J. Path. 38:639, $1961 . \quad 6)$ Schaffner, F. and Popper, H. : Am. J. Path. $38: 393,1961$. 7) Pearse, A.G.F.: Histo chemistry, Boston, Liłtle-Brown, 1960, p. $546 . \quad$ 8) Lison, L. : Histochemie et cytochemie animales, Paris, Gauthier-Villars, 1960. 9) Herxheimer, G. and Tholldte, M., in Henke, F. und Lubarsch, O.: Handbuch der speziellen pathologischen Anatomie und Histologie, Berlin, Springer, 1928, Vol. 5, pt 1, p. $988 . \quad 10)$ Herxheimer, G. und Gerlach, W.: Beitr. path. Anat. 68:93, 1921.

\section{Legends}

Fig. 1 Cholangiolitic hepatitis of probable viral origin, H.E. stain.

Fig. 2 Cholangiolitic hepatitis, SDH visualization. Arrow indicates a reduction of SDH activity in the cells stained with bile pigments. BT:bile thrombus.

Fig. 3 Cholangiolitic hepatitis, mitochondria stain by Regand's methed. Arrow indicates scarcely visible mitochondria in the proliferated ductular cells.

Fig. $4 \mathrm{SDH}$ visualization of the proliferated bile ductules in postnecrotic cirrhosis.

Fig. 5 SDH visualization of the trapped hepatic cells in the connective tissue and a regener- 
ative nodule.

Fig. 6 Reconstructive figure of the proliferated bile ductules in relation to the regenerative nodules, trapped cells in the connective tissue and vascular structure. HA : hepatic artery, PV: portal vein, BD: bile duct, D: bile ductule.

\title{
Studies on the Histogenesis and Function of the Regenerative Nodule in Experimental Rat Cirrhosis III. DNA Content of Regenerative Nodule Cells
}

\author{
Kiyowo Kosaka, Yasuyuki OHTA and Osamu Yokor
}

The Fist Department of Internal Medicine Okayama University Medical School, Okayama

The regenerative nodule in experimental rat cirrhosis induced by a choline deficient diet is defined as a cell mass projected on the surface of the fibrous liver. On microscopic examination ${ }^{1)}$, the cell masses are consisted of a small non-fatty or less fatty cells alined in one cell thick plate. They are the cells of homogenous in the property with reduced succinic dehydrogenase activity ${ }^{2}$. Occasionally another type of the regeneration nodules was also encountered. The nodules are consisted of the cells of not homogeneous in the property. A large cells having a large nuclei with more than two nucleoli are arranged in rather odd fashion. Thus having a structure of adenoma ${ }^{1)}$. Both types of the regenerative nodules formed in fatty liver cirrhosis and proliferate expansively. Customarilly, such newly formed cell mass have been designated as the "regenerative" nodele. However, the mechanism operating the proliferation of the cells has recently debated by some investigators ${ }^{3)}$. A comparative study has been attempted in connection with the study of hepatic cell proliferation after partial hepatectomy in the normal liver by using a cytochemical method", tracing method using radioisotope $\mathrm{P}^{32}{ }^{5)}$, autoradiography using tritiated thymidine ${ }^{6}$, however, no report came out to date for the elucidation of the subject using microspectrophotometry. The results obtained here were based on a study applied a microspectrophotometric method for the determination of DNA content of both the cells of regenerative nodules and internodular areas.

\section{Materials and Methods}

Male, white rats of Sprague strain weighed approximately 100 grams served for the present study. The animals were kept in an individual cage placed in a room of constant temperature and fed a choline deficient diet ${ }^{7)}$ for 206 to 376 days. The five experimental animals reached to the state of nodular cirrhosis were selected for the study. Individual protocoles about the experi- 